\section{The IXth International Congress of Virology}

Glasgow, UK, 8-13 August 1993

The aim of the organizers of the IXth International Congress of Virology (ICV93) of the International Union of Microbiological Societies was 'to bring together a galaxy of distinguished scientists to present their latest data and to review progress over a wide spectrum of virological topics' Indeed, they succeeded in presenting a scientific programme which offered virtually all the important subjects studied in modern virology in a series of plenary sessions, workshops, poster sessions and round tables

A one-day course was organized on the first day of the congress, which was intended to provide an introduction or an update on areas of virology that are current but which may be outside a participant's particular field of expertise, with the objective of providing a background for the plenary sessions and workshops

The plenary sessions dealt with virus structure and assembly expression of viral genomes, molecular basis of disease, virus-host interactions, and the impact of molecular biology on diagnostics and control of virus infections Together these sessions presented an excellent overview of the present state of modern virology, of direct or indirect importance for those working in vaccine-related research fields Vaccine-related topics were more directly addressed in a number of the workshops with their associated poster sessions

The workshops covered an even wider variety of subjects than the plenary sessions, including new developments concerning virus families, control and immunity, diagnosis, pathogenesis, plant virology and phages One workshop directly addressed new trends in vaccine development In several of the studies presented, whole inactivated, split or subunit preparations of viruses were used as candidate vaccines in the presence of a variety of adjuvant systems or immunogenic presentation forms The potential of live viruses attenuated via classical means or via the substitution of selected genes associated with virulence or by reassortment of genes was evaluated Different viral expression systems used to produce viral subunits or virus-like particles were presented, which also were evaluated in association with several adjuvant and presentation systems $A$ separate workshop was entırely devoted to viral expression systems The immunogenicity and protective effect of different recombinant pox viruses and other viruses expressing foreign genes and of chimeric viruses received much attention Most of the immunization studies focused on serum antibody responses and protection, while some also included local IgA, T-helper and cytotoxic T-cell responses Two workshops were entirely devoted to mechanisms of virus neutralization and the importance of cellular immunity to virus infections Studies related to AIDS vaccine development were largely confined to protection experiments and mechanisms of protection induced by subunit vaccines and live attenuated simian immunodeficiency virus (SIV) in SIV macaque models

The two round table sessions about the final steps toward the eradication of smallpox virus and the release of genetically modified viruses and of transgenic virus-resistant hosts attracted a lot of attention from the ICV93 participants and from the lay press

Taken together, the ICV93 was a quite successful meetıng, for which the organizers may be complimented Its abstract book contans a huge amount of information that may provide those working in the field of virus vaccines with many useful 1deas and facts for further consideration

Albert D.M.E. Osterhaus

Department of Virology, Erasmus University Rotterdam, PO Box 1738, 3000 DR Rotterdam, The Netherlands 